\title{
Pancreatic Response to Crystalloid Resuscitation in Experimental Pancreatitis
}

\author{
James A. KnOL, M.D., ${ }^{1}$ Mark G. InMan, M.S., William E. Strodel, M.D., \\ AND FREDERIC E. ECKHAUSER, M.D.
}

Surgery Service, Veterans Administration Medical Center, and Section of General Surgery, Department of Surgery, University of Michigan Medical Center, Ann Arbor, Michigan 48109

Submitted for publication December 9, 1985

\begin{abstract}
Restoration and maintenance of intravascular volume is crucial in acute pancreatitis to prevent hypotension and ensure normal organ perfusion. This study evaluated the hemodynamic and metabolic effects of adequate versus inadequate fluid replacement on the pancreas in a canine model of acute experimental pancreatitis. Bile-trypsin pancreatitis (BTP) was induced in 14 conditioned mongrel dogs. Lactated Ringer's solution was administered intravenously at high (HIR) and low (LIR) infusion rates $(6.5$ and $1.75 \mathrm{ml} / \mathrm{kg} / \mathrm{hr}$, respectively) to 7 dogs each for $4 \mathrm{~h}$. Seven sham-operated controls (CON) received lactated Ringer's at $6.5 \mathrm{ml} / \mathrm{kg} / \mathrm{hr}$ for $3 \mathrm{hr}$. Mean arterial pressure remained unchanged in all groups. Central venous pressure decreased in the LIR group $(P<0.05)$ and remained unchanged in the other groups. Cardiac index fell uniformly $(P<0.05)$ in all groups. Pancreatic blood flow $\left(Q_{\mathrm{P}}\right)$ decreased in the LIR group (73\%) to a significantly greater extent than in the HIR $(23 \%)$ and CON $(8 \%)$ groups, and in the HIR group significantly more than in the CON group. The fall in pancreatic oxygen consumption $\left(\mathrm{O}_{2} C_{\mathrm{P}}\right)$ in both the pancreatitis groups was significant compared to the rise in the CON group. Final changes in $Q_{\mathrm{P}}$ and $\mathrm{O}_{2} C_{\mathrm{P}}$ from baseline were significant only in the LIR group. We conclude that inadequate crystalloid replacement after BTP results in a progressive fall in $Q_{\mathrm{P}}$ and $\mathrm{O}_{2} C_{\mathrm{P}}$. Vigorous fluid replacement incompletely prevents these effects. (C) 1987 Academic Press, Inc.
\end{abstract}

\section{INTRODUCTION}

Vigorous fluid resuscitation is an established aspect of therapy for acute pancreatitis. Early investigators demonstrated blood and plasma volume deficits in patients with severe pancreatitis and showed that the early death rate from shock could be decreased by vigorous fluid replacement [1-3]. The beneficial effects of fluid replacement were initially attributed to normalization of deranged systemic hemodynamics; the effects on the pancreatic circulation were largely ignored. Although the effects of fluid resuscitation on the pancreatic microcirculation during acute pancreatitis have been investigated experimentally, studies have compared crystalloids to colloids or to dextrans and have yielded conflicting results [4-7]. Difficulties

\footnotetext{
${ }^{1}$ To whom correspondence and reprint requests should be addressed at $2920 \mathrm{G}$ Taubman Center, $1500 \mathrm{E}$. Medical Center Drive, Ann Arbor, MI 48109-0331.
}

in comparing the pure volume effects of these different fluids may have contributed to discrepancies observed. Moreover, the rheologic and antithrombotic properties of dextrans may have obscured pure volume effects and complicated interpretation of the results.

This study investigated the effects of high versus low volume crystalloid replacement on the pancreatic microcirculation in acute hemorrhagic pancreatitis. Low volume crystalloid replacement was associated with marked compromise in pancreatic blood flow despite having minimal effect on systemic hemodynamics. High volume fluid replacement had a modulating effect on the fall in pancreatic blood flow associated with acute hemorrhagic pancreatitis, but was unable to maintain flow within the range of the controls. The level of fluid replacement had little effect on the acute depression of pancreatic oxygen consumption associated with acute pancreatitis. 


\section{METHODS}

Twenty-one conditioned mongrel dogs of both sexes weighing 21 to $46 \mathrm{~kg}$ were fasted for $24 \mathrm{~h}$ but allowed water ad libitum. Anesthesia was induced with intravenous pentobarbital sodium $(30 \mathrm{mg} / \mathrm{kg}$ ) and maintained with supplemental doses $(5 \mathrm{mg} / \mathrm{kg}$ ) as needed. Each animal was intubated and ventilated on room air with a Harvard respirator to maintain a normal $P \mathrm{O}_{2}$ and slight hypocarbia. The animal's core temperature was maintained at $38 \pm 0.5^{\circ} \mathrm{C}$ with a heating mattress and heat lamps. A thermistor tip Swan-Ganz catheter was placed for cardiac output determinations, and an additional central venous cannula and an arterial cannula were placed for pressure monitoring. The experimental preparation as well as the methods used for inducing pancreatitis and obtaining hemodynamic and metabolic measurements have been described in detail previously [7].

Through a midline abdominal incision, the body and tail of the pancreas were excised at the pancreatic neck to remove that portion with blood supply from the splenic artery. The posterior pancreaticoduodenal artery and all caudal paraduodenal arterial collateral were ligated to isolate pancreatic arterial inflow to the anterior pancreaticoduodenal artery. An electromagnetic flow probe on the gastroduodenal artery measured blood flow through the anterior pancreaticoduodenal artery to the remaining pancreas. A small cannula was placed into the anterior pancreaticoduodenal vein to sample pancreatic venous effluent for oxygen analysis. Pancreatic oxygen consumption was determined by pancreatic arteriovenous oxygen content difference multiplied by pancreatic blood flow.

A catheter placed retrograde into the right gastroepiploic artery with its tip at the anterior pancreaticoduodenal artery permitted injection of $15-25 \mu \mathrm{m}{ }^{99 m}$ Tc-labeled albumin microsphercs into the pancreatic inflow. A snare on the proximal gastroduodenal artery was tightened during microsphere injection to prevent retrograde flow into the he- patic artery. The proportion of each bolus of microspheres passing through the pancreatic bed into the liver bed was taken as a measurc of percentage arteriovenous shunting [7].

The major pancreatic duct was cannulated external to the duodenum. The duct from the uncinate process was cannulated whenever possible or else ligated at the duodenal wall. Pancreatitis was induced by high-pressure retrograde injection of a bile-trypsin mixture into the pancreatic duct.

During surgical preparation of the model, lactated Ringer's solution was administered iv at $30 \mathrm{ml} / \mathrm{kg} / \mathrm{h}$ to maintain central venous pressure and ensure adequate intravascular volume. The animals were allowed to stabilize for $30 \mathrm{~min}$ following the surgical procedure. Two sets of baseline measurements were then taken at 30-min intervals prior to inducing pancreatitis.

Beginning immediately after induction of pancreatitis, intravenous lactated Ringer's solution was administered continuously over $4 \mathrm{~h}$ to seven dogs at a high infusion rate (HIR) of $6.5 \mathrm{ml} / \mathrm{kg} / \mathrm{h}$ and to seven dogs at a low infusion rate (LIR) of $1.75 \mathrm{ml} / \mathrm{kg} / \mathrm{h}$. Seven additional dogs underwent peripancreatic dissection without induction of pancreatitis $(\mathrm{CON})$ and received lactated Ringer's solution at $6.5 \mathrm{ml} / \mathrm{kg} / \mathrm{h}$ for $3 \mathrm{~h}$. Measurements of systemic and pancreatic hemodynamics, pancreatic oxygen consumption, and pancreatic arteriovenous shunting were obtaincd at 30-min intervals. All animals were sacrificed at the completion of the study. The remaining pancreas was excised and weighed, and samples were prepared for histologic evaluation. All animal care and use in this study followed the guidelines of the Veterans Administration, the American Association for the Accreditation of Laboratory Animal Care, and the Michigan Standard Care Procedures and met the approval of the institution's Animal Use Committee.

Statistical analysis utilized two-way analysis of variance, one-way analysis of variance with comparisons, and where appropriate, paired Student's $t$ tests. Variance is expressed 


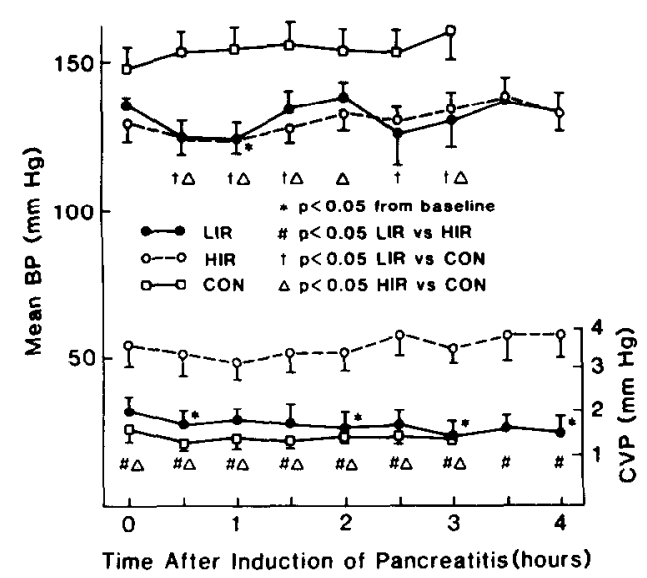

FiG. 1. Mean arterial and central venous pressures following induction of pancreatitis.

as SEM. Only $P$ values less than 0.05 were considered statistically significant.

\section{RESULTS}

All animals survived the experimental period. In HIR and LIR animals, edema and hemorrhage into the pancreas developed consistently along with a marked accumulation of serosanguinous fluid in the peritoneal cavity. These changes in gross morphology were associated with histologic findings consistent with early acute pancreatitis. In contrast, the CON animals developed minimal pancreatic edema only.

Both the experimental and control groups exhibited nearly similar changes in systemic hemodynamics. Mean arterial pressure remained essentially constant over the course of the experiment in all groups (Fig. 1). In the LIR group, a slight but significant decrease in central venous pressure (CVP) from baseline was observed at $4 \mathrm{~h}$ (Fig. 1). Although central venous pressure in the HIR group was consistently higher than in the LIR and CON groups, in neither the HIR or the CON groups did it vary significantly from baseline. Cardiac index fell uniformly and was significantly decreased in all groups at $3 \mathrm{~h}$ (Fig. 2). Partial recovery was observed in the HIR group over the final hour. No significant intergroup differences in cardiac index were observed.

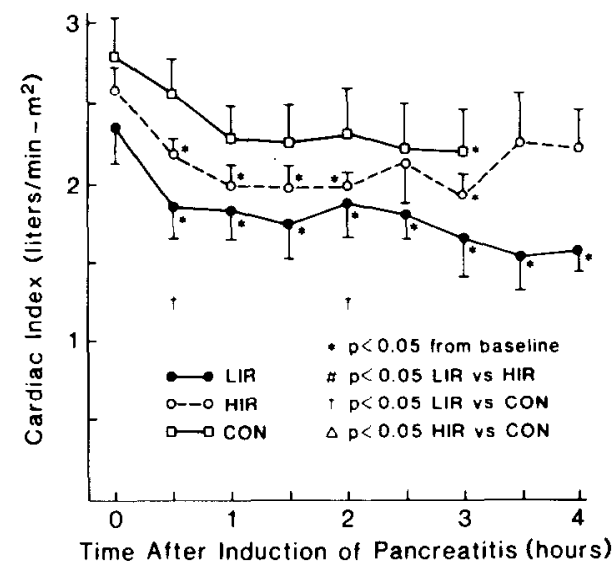

FIG. 2. Cardiac index following induction of pancreatitis.

Marked differences in pancreatic blood flow $\left(Q_{\mathrm{P}}\right)$ occurred between groups (Fig. 3). Four hours following induction of pancreatitis, $Q_{\mathrm{P}}$ in the LIR and HIR groups decreased by 73 and $23 \%$, respectively. By comparison, $Q_{\mathbf{P}}$ in the CON group decreased by only $8 \%$ during the $3-\mathrm{h}$ period of observation. Comparison of fractional changes in $Q_{\mathrm{P}}$ compared to baseline revealed significant differences between all groups (Fig. 4).

Pancreatic arteriovenous shunt flow ( $\left.Q_{\mathrm{Avs}}\right)$ decreased significantly in the LIR group (Fig. 3), although the absolute change was very small. $Q_{\text {Avs }}$ in the CON group was significantly higher than that observed in the

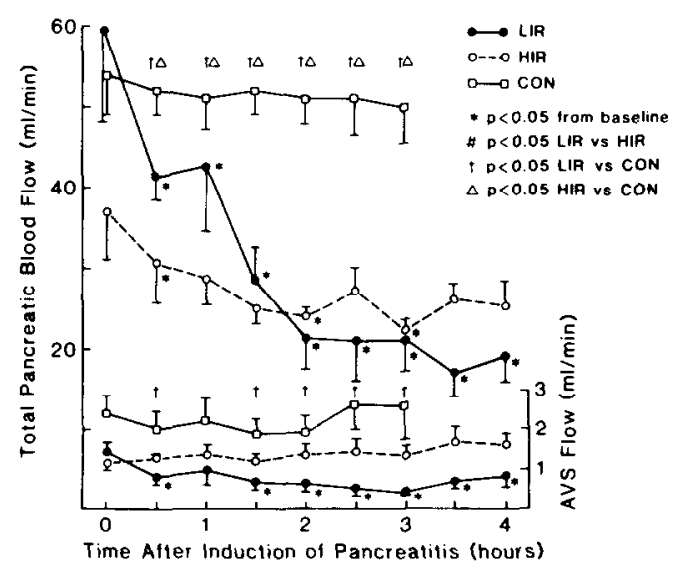

FIG. 3. Total pancreatic blood flow and pancreatic arteriovenous shunt flow following induction of pancreatitis. 


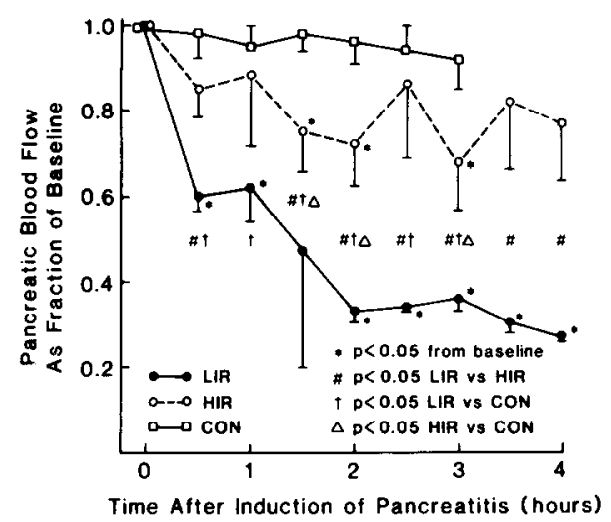

FIG. 4. Total pancreatic blood flow as a fraction of baseline following induction of pancreatitis.

LIR group at $1.5 \mathrm{~h}$ and thercafter. In all groups, $Q_{\mathrm{Avs}}$ accounted for less than $5 \%$ of total pancreatic blood flow.

By $30 \mathrm{~min}$ pancreatic oxygen consumption had decreased by 31 and $28 \%$ in the LIR and HIR groups, respectively. This trend persisted to $3 \mathrm{~h}$ (Fig. 5). Slight recovery toward baseline was seen in the HIR group over the final hour, whereas oxygen consumption continued to decline in the LIR group. Despite final dissimilar trends, differences between these two groups were not significant. In addition, changes in oxygen consumption as a percentage of baseline were not significantly different between the LIR and HIR groups. By comparison, pancreatic oxygen consumption increased in the $\mathrm{CON}$ group, a pattern which differed significantly from that observed with the other two groups.

Correlation analysis of changes in pancreatic oxygen consumption with changes in total pancreatic blood flow was inconclusive. Although the trends observed in $Q_{\mathrm{P}}$ and oxygen consumption over time were similar between the HIR and LIR groups, no statistically significant relationship between these parameters could be demonstrated.

\section{DISCUSSION}

In patients with severe acute pancreatitis, adequate fluid replacement is essential to avoid hypovolemic shock. Because the extent of pancreatic damage is an important determinant of the clinical course and prognosis for ultimate recovery, maintenance of pancreatic blood flow and cellular energy metabolism is important in the early treatment of acute pancreatitis. Experimental trials have demonstrated that survival is increased by high volume fluid replacement, particularly if plasma cxpanders, colloid, or blood are part of the fluid replacement regimen $[1-3,8]$. However, the effects of high volume fluid replacement on the pancreas itself were not studied.

There are no published studies evaluating the role of volume of fluid replacement alone on pancreatic hemodynamics and metabolism during acute pancreatitis. Most studies in which pancreatic and systemic hemodynamic changes have been measured compared the effects of colloid- or dextran-containing fluids to those of saline [4-7]. However, differences in compartment distribution and retention as well as non-volumerelated properties of dextran or colloidal solutions (e.g., antithrombotic, rheologic, or enzyme binding actions) make it difficult to interpret existing data as to the effect of pure volume replacement on the pancreatic microcirculation. Therefore, we chose to assess the effects of fluid administration using a single well-defined isotonic crystalloid solution infused at high and low rates.

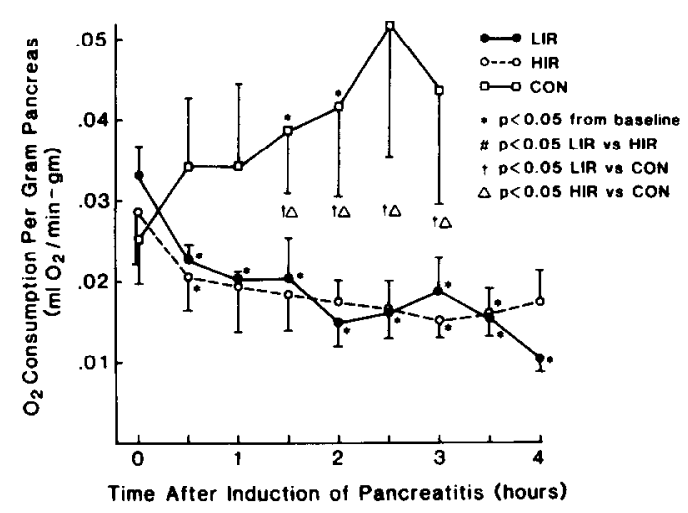

FIG. 5. Pancreatic oxygen consumption following induction of pancreatitis. 
The rate of fluid replacement appeared to affect, of systemic hemodynamic parameters, only central venous pressure. Central venous pressure decreased a small but significant amount in the LIR group but remained unchanged in the other groups. From previous studies using this canine model of hemorrhagic pancreatitis, we determined that maintenance of constant central venous pressure required administration of lactated Ringer's solution at an infusion rate of 6.5 $\mathrm{ml} / \mathrm{kg} / \mathrm{h}$, the infusion rate selected for the HIR group. The decrease in central venous pressure in the LIR group, therefore, was anticipated.

Arterial pressure in all groups remained relatively stable throughout the study, a finding that was unexpected in the LIR group. Donaldson et al., using a similar model of pancreatitis, showed an early marked fall in mean arterial pressure to less than $90 \mathrm{~mm} \mathrm{Hg}$ despite infusion of isotonic fluid at a more rapid rate than that used in our LIR group [4]. The discrepancy between these blood pressure patterns may be explained by the greater volume of fluid administered during the operative preparation of our model. A more recent study of canine pancreatitis from that same laboratory showed that arterial pressure is maintained in animals administered higher volumes of fluid during the preparatory procedure [9].

Cardiac index fell uniformly in all groups. Studley et al. also observed a fall in cardiac index both in control dogs and in those with acute pancreatitis [9]. Barbiturate anesthesia is most likely responsible for the decreases in cardiac index.

While changes in systemic hemodynamics in the LIR group were mild, low volume fluid replacement was associated with major derangements in pancreatic blood flow. Although it has been well documented that $Q_{P}$ is compromised in acute pancreatitis $[4,6,7$, 9-11], results from this study suggest that $Q_{\mathrm{P}}$ is particularly sensitive to decreases in intravascular volume. The $73 \%$ decrease in $Q_{P}$ in the LIR group was significantly greater than the $23 \%$ decrease in the HIR group. In addition, while $Q_{\mathrm{P}}$ continued to decline at $4 \mathrm{~h}$ in the LIR group, $Q_{\mathrm{P}}$ in the HIR group showed evidence of some recovery at $4 \mathrm{~h}$. The difference in $Q_{\mathrm{P}}$ occurred in spite of comparable cardiac indices and mean arterial pressures in the two groups, the only measured systemic difference being the drop in the central venous pressure in the LIR group. It is also noteworthy that high volume fluid repletion alone (HIR group) did not fully prevent a fall in $Q_{\mathrm{P}}$, when compared to the CON group. Our data cannot clarify whether additional fluid increments would completely prevent decreases in $Q_{\mathrm{P}}$ associated with acute pancreatitis. However, it is clear that inadequate fluid replacement even in the absence of shock results in severely compromised $Q_{\mathrm{P}}$ and thereby is likely to hasten progression of pancreatic injury.

Pancreatitis-associated compromise of pancreatic oxygen consumption was not significantly affected by the level of fluid replacement. In the CON group pancreatic oxygen consumption increased despite a slight decrease in $Q_{\mathrm{P}}$. By comparison pancreatic oxygen consumption fell sharply over the first $30 \mathrm{~min}$ in both experimental groups, with no significant differences between the two groups. Other investigators have demonstrated similar decreases in pancreatic oxygen consumption associated with acute pancreatitis [5, 10]. Although it has been suggested that decreases in oxygen consumption may be related directly to changes in blood flow [11], we were unable to confirm such a relationship.

We have previously postulated that decreases in pancreatic oxygen consumption observed in dogs with hemorrhagic pancreatitis may be due to cellular defects in oxygen uptake or utilization associated with the initial insult rather than to decreased pancreatic blood flow [7]. Other studies have shown pancreatitis-associated changes in energy metabolism in the hepatocyte. Those changes include decreases in oxygen consumption, decreases in the respiratory control ratio, a shift from state 3 to state 4 respiration, and ultrastructural damage to hepatic mitochondria [12]. Exposure of hepatocytes to ascitic fluid from animals with experimen- 
tal acute pancreatitis has been reported to cause similar metabolic alterations in hepatic mitochondria [13, 14]. Becker et al. measured metabolic alterations in pancreatic cells after inducing acute pancreatitis in dogs and were able to demonstrate significant decreases in pancreatic ATP and creatine phosphate contents [11].

An early breakdown in pancreatic cellular energy systems similar to that seen in hepatocytes may result in destabilization and enzyme leakage with promotion of cellular necrosis. Although Becker et al. attributed the observed metabolic changes to decreased pancreatic blood flow [11], that contention is not well supported. In a separate study using this same model of acute pancreatitis we have observed a fall in pancreatic oxygen consumption in spite of a marked increase in $Q_{\mathrm{P}}$ induced by glucagon infusion [15]. Altered blood flow is likely to be but one of several mechanisms in the induction of the observed disturbances in energy metabolism.

Early rigorous fluid replacement can usually avert or reverse shock in patients with severe acute pancreatitis. The marked reduction in pancreatic blood flow associated with acute pancreatitis can also be moderated by vigorous fluid resuscitation. However, pancreatic oxygen consumption appears to be less responsive to restoration and maintenance of an adequate intravascular volume. Moreover, no single therapeutic agent has to date been shown to protect against or reverse the abnormality in pancreatic oxygen uptake or utilization associated with acute pancreatitis. Further delineation of this cellular derangement must be sought in developing appropriate treatment for acute pancreatitis.

\section{ACKNOWLEDGMENT}

Computer support was provided by the University of Michigan Computing Fund.

\section{REFERENCES}

1. Elliott, D. W., Zollinger, R. M., Moore, R., and Ellison, E. H. The use of human serum albumin in the management of acute pancreatitis. Gastroenterology 28: $563,1955$.

2. Flliott, D. W. Treatment of acute pancreatitis with albumin and whole blood. Arch. Surg. 75: 573, 1957.

3. Hara, M., Thompson, B. W., and Hudson, L. H. The comparative effects of dextran and blood in lethal sterile hemorrhagic pancreatitis. Surg. Forum 6: $377,1955$.

4. Donaldson, L. A., Williams, R. W., and Schenk, W. G., Jr. Experimental pancreatitis: Effect of plasma and dextran on pancreatic blood flow. Surgery 84: $313,1978$.

5. Donaldson, L. A., and Schenk, W. G., Jr. Experimental acute pancreatitis: The changes in pancreatic oxygen consumption and the effect of dextran 40 . Ann. Surg. 190: 728, 1979.

6. Goodhead, B. Acute pancreatitis and pancreatic blood flow. Surg. Gynecol. Obstet. 129: 331, 1969.

7. Knol, J. A., Edgcomb, L. P., Inman, M. G., and Eckhauser, F. E. Low molecular weight dextran in experimental pancreatitis: Effects on pancreatic microcirculation. J. Surg. Res. 35: 73, 1983.

8. Wright, P. W., and Goodhead, B. The value of dextrans in the treatment of experimental pancreatitis. Surgery 67: 807, 1970.

9. Studley, J. G. N., Lee, J. B., and Schenk, W. G., Jr. Pathophysiology of acute pancreatitis: Evaluation of the effects and mode of action of indomethacin in experimental pancreatitis in dogs. J. Surg. Res. 32: 563, 1982.

10. Becker, H., Senninger, N., Reumont, J., and Vogt, A. Dextran-40 and somatostatin in the treatment of acute experimental pancreatitis. Newsletter: $\mathrm{Na}$ tional Pancreatic Cancer Project 6: 7, 1981. [Abstract]

11. Becker, H., Vinten-Johansen, J., Buckberg, G. D., and Bugyi, H. I. Correlation of pancreatic blood flow and high-energy phosphates during experimental pancreatitis. Eur. Surg. Res. 14: 203, 1982.

12. Pawlicka, J. E., Dlugosz, J., Andrzcjewska, A., and Czabryclewicz, A. The functional and ultra-structural changes of hepatic mitochondria in acute experimental pancreatitis in dogs treated with prostacyclin. Exp. Pathol. 22: 157, 1982.

13. Coticchia, J. M., Lessler, M. A., Ellison, E. C., and Carey, L. C. Mitochondrial dysfunction induced by pancreatitis-associated ascitic fluid. Proc. Soc. Exp. Biol. Med. 172: 412, 1983.

14. Pappas, T. N., Lessler, M. A., Ellison, E. C., and Carey, L. C. Pancreatitis-associated ascitic fluid: Effect on oxygen consumption of liver cells. Proc. Soc. Exp. Biol. Med. 169: 438, 1982.

15. Eckhauser, F. E., Knol, J. A., Inman, M. G., and Strodel, W. E. Efficacy of pharmacologic glucagon in acute experimental pancreatitis. Arch. Surg. 120: $355,1985$. 